\title{
A Strong Form of Yamaguti and Nogi's Stability Theorem for Friedrichs' Scheme*
}

\author{
By \\ Rémi VAILLANCOURT**
}

In this paper we derive the Lax-Nirenberg Theorem [3-4] for Yamaguti and Nogi's pseudo-difference schemes of [7] and, as a corollary, we obtain a strong form of Yamaguti and Nogi's Stability Theorem for Friedrichs' scheme for regularly hyperbolic systems. These are new results.

As in [2] and [7] let $\mathcal{K}$ denote the class of $p \times p$ matrices $k(x, \xi) \in C^{\infty}\left(R_{x}^{n} \times R_{\xi}^{n}-\{0\}\right)$, independent of $x$ for $|x|>R$ fixed, and homogeneous of degree zero in $\xi$.

Lemma (Lax [2]). Every $k \in \mathcal{K}$ can be expanded in a series

$$
k(x, \xi)=\sum_{\alpha} k_{\alpha}(x) e^{i \omega \cdot \xi / \xi \mid},
$$

where $\alpha$ varies over all multi-indices of integers. The series, and the differentiated series, with respect to $x$ or $\xi$, converge uniformly for all $x$, and $|\xi|=1$.

The $h$-family of operators

$$
K_{h} u(x)=\int e^{i x \cdot \xi} k(x, \lambda(h \xi)) \hat{u}(\xi) d \xi
$$

is associated with the symbol $k(x, \lambda(\xi))$ while the Fourier Transform of the adjoint family,

Received May 17, 1969.

Communicated by S. Matsuura.

* The work presented in this paper is supported by the AEC Computing and Applied Mathematics Center, Courant Institute of Mathematical Sciences, New York University, under Contract AT(30-1)-1480 with the U. S. Atomic Energy Commission.

** Department of Mathematics, The University of Chicago. 


$$
\widehat{K_{h}^{*} u}(\xi)=(2 \pi)^{-n} \int e^{-i x \cdot \xi} k^{*}(x, \lambda(h \xi)) u(x) d x
$$

has the symbol $k^{*}\left(x_{1}, \lambda\left(\xi_{2}\right)\right)$. Here the subscripts indicate that the multiplication by the variable $x$ is performed before the differentiation corresponding to the co-variable $\xi$. We write also $k^{* R}(x, \lambda(\xi))$ for $k^{*}\left(x_{1}, \lambda\left(\xi_{2}\right)\right)$.

The Fourier Transform

$$
\hat{k}(\chi, \xi)=(2 \pi)^{-n} \int e^{-i x \cdot x} k(x, \xi) d x
$$

of $k \in \mathcal{K}$ exists and has finite $L^{1}$-maximum norm

$$
\|\hat{k}\|=\int\left[\sup _{\xi}|\hat{k}(\chi, \xi)|\right] d \chi<\infty
$$

and the $L^{2}$ operator norm of $K_{h}$ satisfies the inequality

$$
\left\|K_{h}\right\| \leq\|\hat{k}\|,
$$

provided we let

$$
\hat{k}(\chi, \xi)=\delta(\chi) k(\xi) \quad \text { and } \quad\|\hat{k}\|=\sup _{\xi}|k(\xi)|
$$

for a function $k(\xi)$ independent of $x$. Now, we represent the Fourier Transform of $K_{h} u$ and $K_{h}^{*} u$ in terms of $\hat{k}, k^{*}$ and $\hat{u}$ :

$$
\begin{aligned}
& \widehat{K_{h} u(\xi)}=\int \hat{k}\left(\xi-\xi^{\prime}, \lambda\left(h \xi^{\prime}\right)\right) \hat{u}\left(\xi^{\prime}\right) d \xi^{\prime} \\
& \widehat{K_{h}^{*} u(\xi)}=\int \widehat{k^{*}},\left(\xi-\xi^{\prime}, \lambda(h \xi)\right) \hat{u}\left(\xi^{\prime}\right) d \xi^{\prime} .
\end{aligned}
$$

One sees that $K_{h}$ is associated with the Fourier kernel $\hat{k}=\hat{k}(\chi, \lambda(\xi))$ while $K_{h}^{*}$ is associated with $k^{* R}=\widehat{k^{*}}(\chi, \lambda(\xi+\chi))$. Clearly

$$
\left\|\widehat{k^{* R}}\right\|=\|\hat{k}\| \text {. }
$$

If $k$ is hermitian, $k=k^{*}$, then $K_{h}^{*}$ is associated with $\hat{k}^{R}=\widehat{k}^{* R}$.

Theorem [5]. Suppose that $p \in \mathcal{K}, p(x, \xi) \geq 0$. If $\lambda(\xi) \in C^{2}$, $\lambda(0)=0$, and $\lambda, \lambda_{\xi}$ and $\lambda_{\xi \xi}$ are bounded, then

$$
R e\left\langle P_{h} \Lambda_{h}^{2} u\right\rangle \geq-K h\|u\|^{2}, u \in L_{x}^{2},
$$

for all $h$ and some constant $K$. 
This particular form of the Lax-Nirenberg theorem is a sharp form of Theorem 3, [7], p. 159; in fact the proof of the latter requires that $p$ be positive definite, $p>0$.

Corollary [5]. If $\lambda=k / h$ satisfies

$$
\lambda \leq \frac{1}{\sqrt{n} \mu_{0}},
$$

then Friedrichs' scheme

$$
S_{h} u=\sum_{j=1}^{n}\left\{\frac{u\left(x+\delta_{j}, t\right)+u\left(x-\delta_{j}, t\right)}{2 n}+\lambda A_{j}(x) \frac{u\left(x+\delta_{j}, t\right)-u\left(x-\delta_{j}, t\right)}{2}\right\}
$$

is stable in the sense of Lax-Richtmyer.

Here $\mu_{0}$ is the supremum of the spectral radius of the regularly hyperbolic matrix $\sum a_{j}(x) \xi_{j}$ over $|\xi|=1$ and all $x \in R_{x}^{n}$.

This corollary is a strong form of Theorem ${ }^{1)} 4,[7]$, p. 162. The latter had only strict inequality in (1.2).

Our corollary follows from the proof of Theorem 4 [7], pp. 162165 , if in the last step of the proof one applies our theorem instead of Theorem 3 [7]. Therefore we need only prove our theorem.

We adapt to the case at hand Friedrichs' proof $[1,6]$ of the Lax-Nirenberg theorem for pseudo-differential operators.

Choose a smooth even mollifier $q^{2}(\sigma)$ with support in the unit sphere, $|\sigma| \leq 1$, and integral 1 ,

$$
\int q^{2}(\sigma) d \sigma=1
$$

Convolve

$$
g(x, \xi)=p(x, \lambda(\xi))|\lambda(\xi)|^{2}
$$

with $q^{2}$ to obtain the mollified symbol

$$
a(x, \xi)=\int g\left(x, \xi-h^{1 / 2} \sigma\right) q^{2}(\sigma) d \sigma,
$$

which, after the change of variable

$$
\zeta=\xi-h^{1 / 2} \sigma,
$$

1) Theorem " 3 " is a misprint in [7], p. 162 . 
becomes

$$
a(x, \xi)=h^{-n / 2} \int g(x, \zeta) q^{2}\left(h^{-1 / 2}[\xi-\zeta]\right) d \zeta .
$$

Rearrange (1.7) into the double symbol

$$
\begin{aligned}
b\left(\xi_{2}, x_{1}, \xi_{1}\right)=h^{-n / 2} \int & q\left(h^{-1 / 2}\left[\xi_{2}-\zeta\right]\right) g\left(x_{1}, \zeta\right) \\
\cdot & q\left(h^{-1 / 2}\left[\xi_{1}-\zeta\right]\right) d \zeta .
\end{aligned}
$$

Obviously $b$ generates the symmetric operator

$$
\widehat{B_{h} u(\xi)}=\int \hat{b}\left(h \xi, \xi-\xi^{\prime}, h \xi^{\prime}\right) \hat{u}\left(\xi^{\prime}\right) d \xi^{\prime} .
$$

We complete the proof by means of three lemmas.

Lemma 1.1. $\left\langle B_{h} u, u\right\rangle \geq 0, u \in L_{x}^{2}$.

Lemma 1.2. $\left\|A_{h}-G_{h}\right\|=0(h)$.

Lemma 1.3. $\left\|B_{h}-S y A_{h}\right\|=0(h)$.

This yields the desired result:

$$
\begin{aligned}
-R e\left\langle P_{h} \Lambda^{2} u, u\right\rangle & \leq\left\langle\left(B_{h}-S y G_{h}\right) u, u\right\rangle \\
& \leq\left[\left\|B_{h}-S y A_{h}\right\|+\left\|A_{h}-G_{h}\right\|\right]\|u\|^{2} \leq 0(h)\|u\|^{2} .
\end{aligned}
$$

Proof of Lemma 1.1. Since $b\left(h \xi_{2}, x_{0}, h \xi_{1}\right)$ is a non-negative symmetric kernel for each value $x_{0}$ of $x$ :

$$
\iint \overline{\hat{u}}\left(\xi_{2}\right) e^{i \xi_{2} \cdot x_{0}} b\left(h \xi_{2}, x_{0}, h \xi_{1}\right) \hat{u}\left(\xi_{1}\right) e^{-i \xi_{1} \cdot x_{0}} d \xi_{1} d \xi_{2} \geq 0,
$$

integrate with respect to $x_{0}$, change the order of integration and apply Parserval's relation to get

$$
0 \leq \iint \overline{\hat{u}}\left(\xi_{2}\right) \hat{b}\left(h \xi_{2}, \xi_{2}-\xi_{1}, h \xi_{1}\right) \hat{u}\left(\xi_{1}\right) d \xi_{1} d \xi_{2}=\left\langle B_{h} u, u\right\rangle \text {. }
$$

Proof of Lemma 1.2. By (1.3) and (1.5),

$$
\hat{a}(\chi, \xi)-\hat{g}(\chi, \xi)=\int\left[\hat{g}\left(\chi, \xi-h^{1 / 2} \sigma\right)-\hat{g}(\chi, \xi)\right] q^{2}(\sigma) d \sigma .
$$

To find a bound for $|\hat{a}-\hat{g}|$ note that $\hat{g}_{\xi_{\mu}}(\chi, \xi)$ exists and is uniformly Lipschitz continuous in $\xi$ with Lipschitz bound $\hat{k}_{\mu}(\chi) \in L^{1}$. This follows from the representation (1.1) for $p(\chi, \xi)$ and the conditions on $\lambda$. A Taylor expansion of $\hat{g}\left(\chi, \xi-h^{1 / 2} \sigma\right)-\hat{g}(\chi, \xi)$ in $h^{1 / 2} \sigma$ yields 
the estimate

$$
\begin{aligned}
|\hat{a}(\chi, \xi)-\hat{g}(\chi, \xi)| & \leq h^{1 / 2}\left|\sum_{\mu} \hat{g}_{\xi \mu}(\chi, \xi) \int \sigma_{\mu} q^{2}(\sigma) d \sigma\right| \\
& +h \sum_{\mu} \hat{k}_{\mu}(\chi) \int|\sigma|^{2} q^{2}(\sigma) d \sigma
\end{aligned}
$$

The term involving $h^{1 / 2}$ is zero since $q^{2}$ is even. Since $\int|\sigma|^{2} q^{2}(\sigma) d \sigma \leq 1$, and $\Sigma \int \widehat{k_{\mu}}(\chi) d \chi<\infty$, Lemma 1.2 follows.

Proof of Lemma 1.3. By (1.7), (1.8) and (1.6) $A_{h}+A_{h}^{*}-2 B_{h}$ is associated with

$$
\begin{aligned}
& \hat{a}(\chi, h \xi)+\hat{a}(\chi, h \xi+h \chi)-2 \hat{b}(h \xi+h \chi, \chi, h \xi) \\
& \quad=\int \hat{g}\left(\chi, h \xi-h^{1 / 2} \sigma\right)\left[q\left(\sigma+h^{1 / 2} \chi\right)-q(\sigma)\right]^{2} d \sigma
\end{aligned}
$$

Thus,

$$
\begin{aligned}
\left|\hat{a}+\hat{a}^{R}-2 \hat{b}\right| & \leq \sup _{\xi}|\hat{g}(\chi, \xi)||\chi|^{2} h\left[\sum \iint_{\mu}^{1}\left|\partial_{0} q\left(\sigma+\beta h^{1 / 2} \chi\right)\right| d \beta d \sigma\right]^{2} \\
& =C h \sup _{\xi}|\hat{g}(\chi, \xi)| \mid \chi_{\left.\right|^{2}} .
\end{aligned}
$$

Integration with respect to $\chi$ yields Lemma 1.3 :

$$
\left\|\hat{a}+\hat{a}^{R}-2 \hat{b}\right\|=0(h) .
$$

This completes the proof of the theorem.

\section{References}

[1] Friedrichs, K. O., Pseudo-Differential Operators, An Introduction, Lecture notes with the assistance of R. Vaillancourt, Courant Inst. Math. Sci., New York Univ., 1968, 208 pp.

[2] Lax, P. D., The $L_{2}$ operator calculus of Mikhlin, Calderón and Zygmund, Mimeographed lecture note, Courant Inst. of Math. Sci., New York Univ., 1963, $17 \mathrm{pp}$.

[3] Lax, P. D. and L. Nirenberg, On stability for difference schemes: a sharp form of Gårding's inequality, Comm. Pure Appl. Math. 19 (1966), 473-492.

[4] - A sharp inequality for pseudo-differential and difference operators, Proc. Symp. on Singular Integrals, Amer. Math. Soc. 10 (1967), 213-217.

[5] Vaillancourt, R., Pseudo-Translation Operators, Thesis, New York Univ., 1969, $113 \mathrm{pp}$.

[6] - A simple proof of Lax-Nirenberg Theorems, Comm. Pure. Appl. Math., to appear.

[7] Yamaguti, M. and T. Nogi, An algebra of pseudo-difference schemes and its application, Publ. RIMS Kyoto Univ. Ser. A, 3 (1967), 151-166. 
